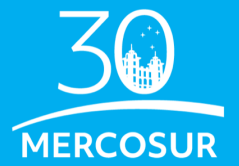

$$
\begin{array}{c|l}
++ & \text { TPR } \\
+ & \begin{array}{l}
\text { Tribunal Permanente } \\
\text { de Revisión }
\end{array}
\end{array}
$$

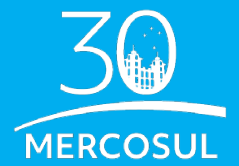

\title{
Serie Jurisprudencia LAUDOS $N^{\circ} 2$, Mayo 2021
}

Laudo $N^{\circ}$ 01/2006

Laudo complementario del Tribunal Permanente de Revisión que resuelve el recurso de aclaratoria interpuesto por la República Argentina en relación al laudo arbitral dictado por este ente el 20 de diciembre de 2005 en la controversia "prohibición de importación de neumáticos remoldeados procedentes del Uruguay"
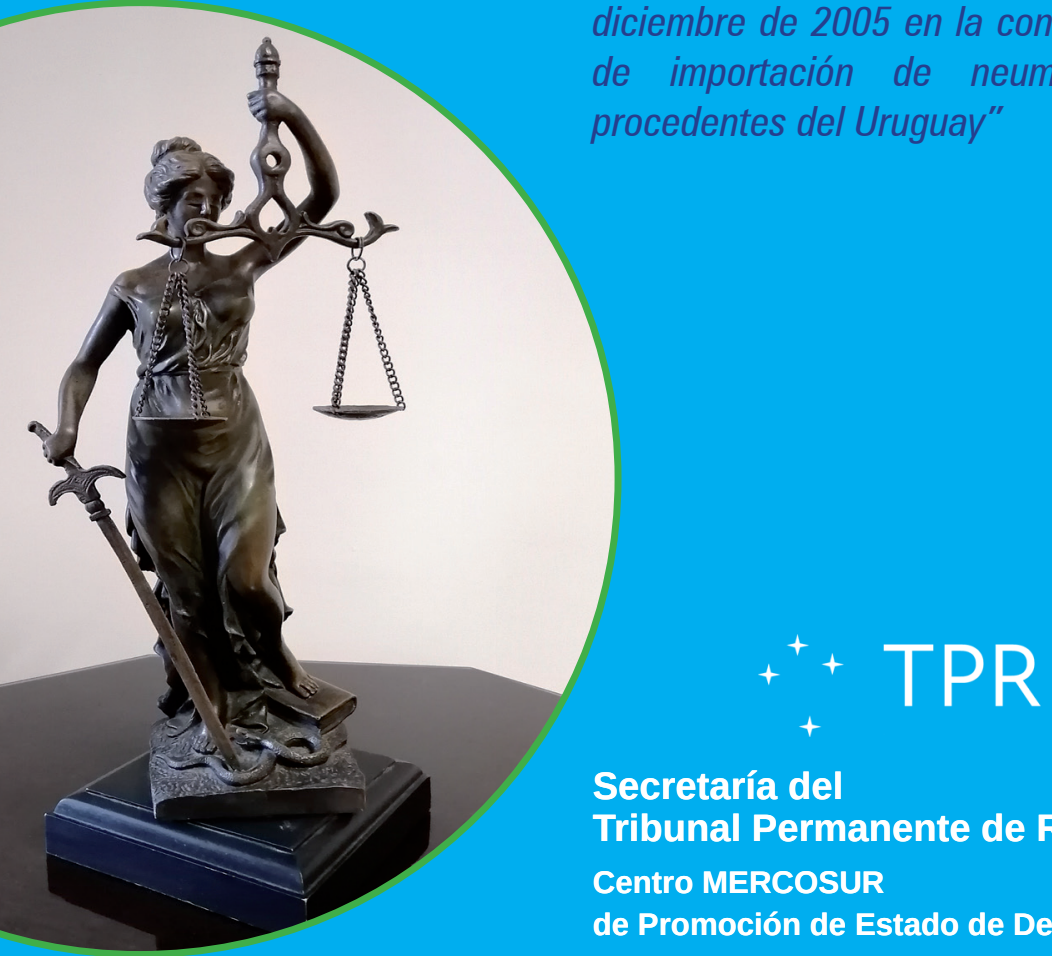

Secretaría del Tribunal Permanente de Revisión Centro MERCOSUR de Promoción de Estado de Derecho 


\section{Serie: Jurisprudencia * LAUDOS}

\section{N. ${ }^{\circ} 2$}

Secretaría del Tribunal Permanente de Revisión

Equipo de trabajo:

Coordinador: Juan Manuel Rivero Godoy

Colaboradores:

Brenda Maffei

Maider Méndez

Renata Cenedesi

Manuel Fernández

Secretaría del Tribunal Permanente de Revisión

Centro Mercosur de Promoción de Derecho 


\section{FICHA CATALOGRÁFICA}

\section{Clasificación. 341.2458 Secretaría del Tribunal Permanente de Revisión \\ SE446 I Centro Mercosur de Promoción de Estado de Derecho}

LAUDO N.ㅇ 01/2006: Laudo complementario del Tribunal Permanente de Revisión que resuelve el recurso de aclaratoria interpuesto por la República Argentina en relación al laudo arbitral dictado por este ente el $\mathbf{2 0}$ de diciembre de 2005 en la controversia "prohibición de importación de neumáticos remoldeados procedentes del Uruguay/Centro Mercosur de Promoción de Estado de Derecho; Ed. Asunción: Secretaría del Tribunal Permanente de Revisión. 2021

14 p.: 22,4 x 15,4 cm. (Laudos no1)

ISSN:

1. Derecho internacional. 2. Integración regional. 3. Arbitraje internacional. 4. Mercosur. 5. Derechos económicos. I. Título. II. Autor

Bajo términos de licencia Creative commons 4.0

Secretaría del Tribunal Permanente de Revisión. Centro MERCOSUR de Promoción de Estado de Derecho.

Asunción, República del Paraguay, 2021

La reproducción total o parcial de esta publicación es autorizada siempre que se cite la fuente.

La información contenida en la publicación es responsabilidad exclusiva del autor/es de la misma. 


\section{Serie: Jurisprudencia * LAUDOS \\ N. ${ }^{\circ} 2$}

\section{LAUDO N. ${ }^{\circ} 01 / 2006$}

Laudo complementario del Tribunal Permanente de Revisión que resuelve el recurso de aclaratoria interpuesto por la República Argentina en relación al laudo arbitral dictado por este ente el 20 de diciembre de 2005 en la controversia "prohibición de importación de neumáticos remoldeados procedentes del Uruguay

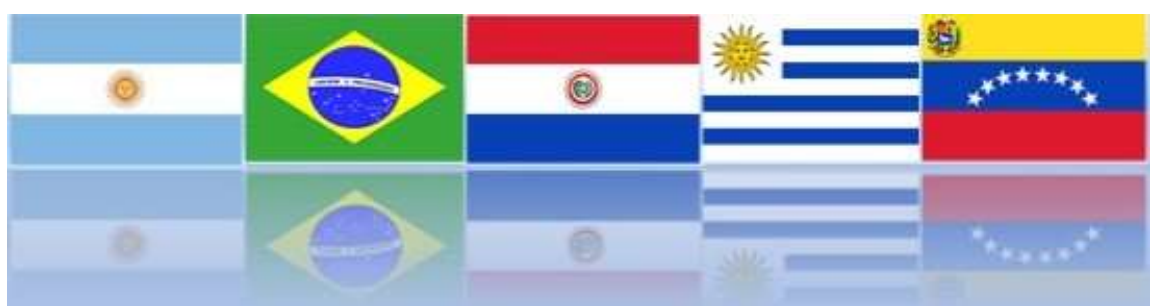

Asunción, mayo 2021 


\section{Contenido}

1. INTRODUCCIÓN..................................................... 1

2. PRESENTACIÓN Y RESUMEN GENERAL DEL

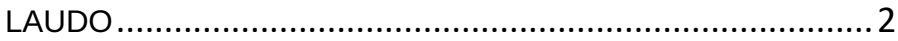

2.1. Ficha técnica....................................................... 2

2.2. Presentación........................................................ 4

2.3. Resumen técnico jurídico del Laudo ..................... 5

3. REFERENCIAS BIBLIOGRÁFICAS .......................10

4. GUÍA DE BIBLIOGRAFÍA COMPLEMENTARIA ........11 



\section{Serie: Jurisprudencia * Laudos $\mathrm{N}^{0} 2$}

\section{Introducción}

La Secretaría del Tribunal Permanente de Revisión presenta una nueva publicación, un resumen técnicojurídico de la serie Laudos relacionada al segundo laudo emitido por el TPR, en el que se observa la continuidad del caso planteado por la República Oriental del Uruguay en el año 2005, sobre la aplicación de una ley de la República Argentina en la importación de neumáticos remoldeados y que afecta los intereses de un país integrante del Mercosur.

En esta oportunidad es la República Argentina quién plantea ante el TPR, treinta y un puntos para ser clarificados sobre lo dictaminado en el primer laudo del Tribunal Permanente de Revisión.

Este nuevo número elaborado en conjunto por el Centro Mercosur de Promoción de Estado de Derecho (CMPED), Área Jurídica, Biblioteca y Archivo de Documentos, Secretaría y Administración e Informática y Base de Datos de la ST, es una publicación en que el CMPED realiza como principal responsable en cumplimiento de sus funciones ${ }^{1}$.

\footnotetext{
${ }^{1}$ 1. Trabajos de investigación relacionados con la promoción del Estado de Derecho, democracia, Derechos Humanos y libertades fundamentales en los procesos de integración regional, con énfasis en el mecanismo de solución de controversias del Mercosur.

2. Difusión, a través de la realización de cursos, conferencias, seminarios, foros, publicaciones, reuniones de académicos, representantes gubernamentales y representantes de la sociedad civil.

3. Cursos de capacitación, programas de intercambio, oferta de becas de estudio dirigidas a profesionales, en función de su presupuesto y convenios que faciliten estas actividades.
} 


\section{Presentación y resumen general del laudo}

\subsection{Ficha técnica}

LAUDO: Segundo laudo emitido por el Tribunal Permanente de Revisión planteando ante el TPR a través de un recurso de aclaratoria del laudo emitido por el TPR, caratulado "Recurso de Revisión contra el Laudo del TAH en la controversia "Prohibición de importación de neumáticos remoldeados procedentes del Uruguay".

FECHA DE INTERPOSICIÓN DEL RECURSO: 05/01/2006

FECHA DE EMISIÓN DEL LAUDO: 13/1/2006

PARTES:

RECURRENTE: República Argentina

ÁRBITROS: Dr. Nicolás Eduardo Becerra, por Argentina, Dr. Ricardo Olivera, por Uruguay, y Dr.

4. Un espacio en la página web del TPR, para promocionar los trabajos, convocatorias, cursos, seminarios, congresos, etc.

5. Un espacio especializado destinado a publicaciones diversas que deriven del CMPED dentro de la biblioteca de la STPR, como también, la adquisición de bibliografía específica en temas relacionados con promoción del Estado de Derecho, democracia, Derechos Humanos y libertades fundamentales en los procesos de integración regional, arbitraje, solución de controversias; con énfasis en el Mercosur. 
Wilfrido Fernández de Brix de Paraguay como presidente.

NORMATIVA APLICADA EN EL LAUDO:

- Artículo 34 Protocolo de Olivos ${ }^{2}$

- Artículo 28 de Protocolo de Olivos ${ }^{3}$

Palabras clave: Arbitraje internacional, recurso de aclaratoria, Laudos, Tribunal Permanente de Revisión, Derechos económicos, Libertad de comercio, Mercosur, Argentina.

2 El cual establece: Derecho aplicable 1. Los Tribunales Arbitrales Ad Hoc y el Tribunal Permanente de Revisión decidirán la controversia en base al Tratado de Asunción, al Protocolo de Ouro Preto, a los protocolos y acuerdos celebrados en el marco del Tratado de Asunción, a las Decisiones del Consejo del Mercado Común, a las Resoluciones del Grupo Mercado Común y a las Directivas de la Comisión de Comercio del Mercosur, así como a los principios y disposiciones de Derecho Internacional aplicables a la materia. 2. La presente disposición no restringe la facultad de los Tribunales Arbitrales Ad Hoc o la del Tribunal Permanente de Revisión cuando actúe en instancia directa y única, conforme a lo dispuesto en el artículo 23 de decidir la controversia ex aequo et bono, si las partes así lo acordaren. A partir de la entrada en vigencia del Protocolo Modificatorio de Olivos, su contenido forma parte del Protocolo de Olivos y por lo tanto los países que se adhieran al proceso de integración por el Tratado de Asunción se adhieren ipso iure al Protocolo de Olivos modificado por el mencionado protocolo modificatorio.

${ }^{3}$ Recurso de aclaratoria 1. Cualquiera de los Estados partes en la controversia podrá solicitar una aclaración del laudo del Tribunal Arbitral Ad Hoc o del Tribunal Permanente de Revisión y sobre la forma en que el laudo deberá cumplirse, dentro de los quince (15) días siguientes a su notificación. 2. El Tribunal respectivo se expedirá sobre el recurso dentro de los quince (15) días siguientes a la presentación de dicha solicitud y podrá otorgar un plazo adicional para el cumplimiento del laudo. 


\subsection{Presentación}

En esta oportunidad se realiza un resumen sobre el Laudo $\mathrm{N}^{\circ}$ 1/2006 relativo al recurso de aclaratoria interpuesto por la República Argentina contra el Laudo 1/2005 relativo a la "Prohibición de Importación de Neumáticos Remoldeados Procedentes del Uruguay".

Como fue indicado en el resumen del Laudo del TPR 1/2005, la controversia suscitada por la prohibición de importación de neumáticos remoldeados puede ser considerada, debido a sus diversas aristas, como un "leading case". En este sentido se remarca debido al conflicto que generó entre los Estados y los intereses creados en torno a él, algunos autores, lo denominaron: "la guerra de los neumáticos". Esto porque, además de la oposición de diferentes principios, como el libre comercio y la protección del medio ambiente que se derivaron de la controversia, el caso también significó una disputa entre diferentes Estados y, al interior de cada Estado, un conflicto entre las industrias de remodelación y de fabricación de neumáticos nuevos, cada uno defendiendo sus propios y contrapuestos intereses económicos ${ }^{4}$.

\footnotetext{
${ }^{4}$ MAFFEI, Brenda. Análisis sistémico de la controversia por los neumáticos remoldeados: un caso emblemático a 15 años de conformación del Tribunal Permanente de Revisión. Revista de la Secretaría del Tribunal Permanente de Revisión, 2019, Año 7, n 14, p. $150 . \quad$ Disponible en: 


\section{Serie: Jurisprudencia * Laudos $\mathrm{N}^{0} 2$}

Desde un punto de vista eminentemente procesal, también, es un caso de relevancia, ya que fueron utilizadas todas las instancias establecidas en el Protocolo de Olivos. En esta oportunidad lo que se analiza es el Recurso de aclaratoria, como fue indicado anteriormente.

La República Argentina plantea 31 ítems sobre los cuales solicita ser clarificados por el Tribunal. En este resumen se organizan, para una mejor comprensión se analizar estos ítems de acuerdo a la temática planteada, como son: el alcance del Recurso de aclaratoria, la distinción entre cuestiones de hecho y de derecho, sobre la excepción al principio de libre comercio, sobre el carácter vinculante del antecedente jurisprudencial, sobre la inversión de la carga de la prueba y sobre el traslado a terceros países no partes en la controversia y la labor del TPR.

\subsection{Resumen técnico jurídico del Laudo}

\section{Cuestiones analizadas:}

1) Alcance del Recurso de aclaratoria

Según la interpretación del TPR, el recurso de aclaratoria debe tener por objeto: a) la corrección de un error material, b) la aclaración de cualquier expresión oscura, sin desde luego alterar lo sustancial de la decisión objeto del recurso, c) suplir alguna omisión en la que el TPR hubiera incurrido en relación a cualquier pretensión deducida y discutida en el litigio. En el numeral XXV del Laudo, el TPR sostiene que no puede actuar como órgano de consulta cuando actúa en la 


\section{Serie: Jurisprudencia * Laudos $\mathrm{N}^{0} 2$}

modalidad de recurso de revisión, y mucho menos con posterioridad a haber emitido el laudo arbitral en cuestión.

El TPR argumenta que la República Argentina ha usado en gran parte el recurso de aclaratoria con la intención sustancial de reabrir la instancia y el debate acerca de las cuestiones decididas por el referido laudo arbitral. Para clarificar la cuestión, el Tribunal cita a Alfredo Antezana Palacios, quien indica que: "Por concepto oscuro, se entiende cualquier discordancia que resulte entre la idea y los vocablos entrelazados para representarla; es una cuestión puramente idiomática que el Juez deberá examinar con cuidado, a fin de evitar abusos en el recurso de aclaratoria. Si los términos son lo suficientemente claros, no deberá explicarlos ni insistir sobre ellos, sino que se deberá limitar a decir no ha lugar en virtud de esa claridad. Es importante no confundir la oscuridad con la equivocación. El recurso de aclaratoria no puede servir para encubrir una reposición totalmente improcedente. Sin que sea procedente renovar el debate sobre la interpretación y aplicación de las leyes, doctrina y jurisprudencia hechas por un tribunal al fallar el asunto.

2) Distinción entre cuestiones de hecho y de derecho

El TPR mantiene la tesitura de que sería un anacronismo no mantener la distinción entre cuestiones de derecho y, de hecho. En este sentido, el TPR ha cumplido su rol institucional en tal sentido. En el punto XXIII el TPR sostiene que se definen como cuestiones de derecho el análisis de cuestiones fácticas en el caso de manifiesta arbitrariedad e irrazonabilidad. 


\section{3) Sobre la excepción al principio de libre comercio}

Con relación al principio de libre comercio el TPR vuelve a identificar la distinción entre el principio de libre comercio vis a vis la invocación de una excepción medio-ambiental, argumentando que el Tribunal no tuvo como objetivo ignorar las distintas modalidades de la integración ni pretendió directa o indirectamente limitar el objetivo del Mercosur a un mero intercambio comercial o un acceso preferencial de mercaderías entre los Estado-parte. Asimismo, el TPR sostiene que de manera alguna se pretendió soslayar cuerpo normativo alguno vigente en el Mercosur, y mucho menos al Acuerdo marco sobre Medio Ambiente del Mercosur, el cual lamentablemente por ser un acuerdo marco no contiene ninguna norma sobre los criterios de rigor a ser utilizado por un Tribunal para evaluar si una medida en cuestión deviene o no procedente para restringir el libre comercio dentro de un esquema de integración. En este sentido, ni el preámbulo del Tratado de Asunción, ni cualquier parte de este Tratado aportan algo en relación al vacío normativo reseñado por el TPR. Ni siquiera haría falta ninguna jurisprudencia para sostener en cuanto al primer criterio de rigor que la medida analizada era restrictiva del libre comercio. Resulta obvio que una prohibición de importación es una restricción al libre comercio. Resulta ser un concepto auto explicativo.

EI TPR deja constancia de que el concepto de discriminación no se altera en el caso de una excepción medio-ambiental. Reitera, además, que en la exposición de motivos de la Ley argentina en estudio se aludía textualmente a la protección de la industria nacional, además de otros dos factores (seguridad en 


\section{Serie: Jurisprudencia * Laudos $\mathrm{N}^{0} 2$}

la vía pública y protección del medio ambiente). En un esquema de integración, de la lectura literal del texto de esta exposición de motivos, ya queda descartada inlimine dicha justificación.

La tesis argentina, señala el TPR, consiste en afirmar que la única proporcionalidad aceptable es la prohibición de ingreso del producto a territorio nacional, pero la misma, indica el TPR, no tiene asidero jurídico alguno. Los árbitros sostienen que, dadas las precedentes consideraciones en casos como el de autos, no es fácil pero no es imposible, realizar una estimación o medición de la proporcionalidad. El deber constitucional resaltado por la representación argentina obviamente obliga a los tomadores de decisión a disponer responsablemente las medidas del caso, pero de ahí a pretender concluir que existe un deber constitucional de directamente prohibir la importación hay demasiada e insalvable distancia.

4) Sobre el carácter vinculante del antecedente jurisprudencial

Al respecto de esta cuestión, el TPR, en el punto $\mathrm{VI}$ del recurso de aclaratoria, asevera que resulta obvio que no le puede ser vinculante ningún antecedente jurisprudencial sentado por ningún Tribunal del Mercosur, mucho menos uno sentado por Tribunal Arbitral Ad hoc.

5) Sobre la inversión de la carga de la prueba

Al respecto de la inversión de la carga de la prueba, el TPR sostiene que es una cuestión jurídica que solo puede ser hecha por un juzgador cuando está autorizado por ley. Confirma su disconformidad con la inversión de la carga de la prueba. El laudo arbitral del 


\section{Serie: Jurisprudencia * Laudos $\mathrm{N}^{0} 2$}

TAH tiene además una doble incongruencia: por un lado, invierte la carga de la prueba y por otro lado analiza las pruebas en base a principios que no corresponden al caso.

6) Sobre el traslado a terceros países no partes en la controversia y la labor del TPR

Con relación al traslado a terceros países lo más relevante a resaltar de este punto es la opinión del TPR que indica que si se acoge a la tesis argentina, tendríamos la incongruencia de que del Tribunal sólo puede establecer o determinar algo en ejercicio de su potestad jurisdiccional cuando haya una norma que expresamente le faculte, cuando que lo correcto es precisamente lo contrario: solamente está impedido de algo cuando existe una norma que expresamente le prohíba hacerlo, a mayor abundamiento, la experiencia histórica del derecho de integración y del derecho comunitario, nos demuestra que inclusive la mayor parte de las características fundamentales de tal derecho fueron originariamente elaboraciones jurisprudenciales en razón de la proficua labor institucional realizada por los tribunales en cuestión cumpliendo su responsabilidad histórica e institucional dentro de un proceso de integración.

\section{7) Decisión}

Finalmente, el TPR decide:

Se presentó Recurso de Aclaratoria sobre 31 ítems. Luego de un análisis pormenorizado de los ítems se hizo una consideración adicional por la cual el TPR interpretó que Argentina intentaba reabrir el debate.

Decisión: 1) Por mayoría, No hacer lugar al Recurso de Aclaratoria presentado por Argentina. 2) Por 


\section{Serie: Jurisprudencia * Laudos $\mathrm{N}^{0} 2$}

unanimidad, Disponer la notificación por correo privado a las partes. 3) Por unanimidad, disponer la notificación al mero efecto informativo a la República Federativa de Brasil y a la República de Paraguay, así como a la Secretaría del Mercosur. 4) Por unanimidad disponer la traducción inmediata al portugués del presente laudo, con la constancia de que la versión en español será siempre la única oficial y prevalecerá sobre la versión del mismo traducida al portugués. En razón del carácter no oficial de la misma, la referida versión en portugués no será autenticada por los árbitros actuantes del TPR. 5) Regístrese, notifíquese en forma inmediata y publíquese.

\section{Referencias bibliográficas}

ESPASA CALPE. Diccionario jurídico Espasa. Madrid: Espasa Calpe, 2004

MERCOSUR. "Protocolo de Brasilia"

MERCOSUR. "Protocolo de Olivos para la Solución de Controversias en el MERCOSUR"

MERCOSUR. "Protocolo de Ouro Preto"

MERCOSUR. "Tratado de Asunción

MERCOSUR. "Protocolo Modificatorio del Protocolo de Olivos"

MERCOSUR. MERCOSUR/CMC/DEC N N37/03: Reglamento del Protocolo de Olivos 


\section{Serie: Jurisprudencia * Laudos $\mathrm{N}^{0} 2$}

MERCOSUR. MERCOSUR/CMC/DEC No30/05: Reglas de procedimiento del TPR

TRIBUNAL PERMANENTE DE REVISIÓN. Recurso de revisión presentado por la República Oriental del Uruguay contra el laudo arbitral del tribunal arbitral ad hoc del 25 de octubre de 2005 en la controversia "prohibición de importación de neumáticos remoldeados procedentes del Uruguay" disponible en:

https://www.tprmercosur.org/es/docum/laudos/Laudo 012005 es.pdf

TRIBUNAL PERMANENTE DE REVISIÓN. "Laudo complementario del Tribunal Permanente de Revisión que resuelve el recurso de aclaratoria interpuesto por la república argentina en relación al laudo arbitral dictado por este ente el 20 de diciembre de 2005 en la controversia "prohibición de importación de neumáticos remoldeados procedentes del Uruguay", 2006. Disponible en: https://www.tprmercosur.org/es/docum/laudos/Laudo 012006 es.pdf

\section{Guía de bibliografía complementaria}

BERTONI, Liliana. Laudos arbitrales en el Mercosur. Buenos Aires. Ciudad Argentina, 2006

DREYZIN DE KLOR, Adriana. "Comercio en el Mercosur y desarrollo: límites. A propósito del laudo once y primer laudo del TPR". Boletín Mexicano de Derecho Comparado. Número conmemorativo, p. 211244. 


\section{Serie: Jurisprudencia * Laudos $\mathbf{N}^{0} 2$}

https://revistas.juridicas.unam.mx/index.php/derechocomparado/article/view/4054/5197

GIORGIERI, María Paula. "Solución de controversias en el Mercosur: Evolución de los regímenes y su aplicación" (Tesis). Orientador: Julio Berlinski. Universidad Torcuato di Tella. Buenos Aires, 2013.Disponible

en: https://repositorio.utdt.edu/bitstream/handle/utdt/1649/ LEI 2013 Giorgieri.pdf?sequence=1\&isAllowed=y

MAFFEI, Brenda. "Análisis sistémico de la controversia por los neumáticos remoldeados: un caso emblemático a 15 años de conformación del Tribunal Permanente de Revisión". Revista de la Secretaría del Tribunal Permanente de Revisión, 2019, Año 7, n 14, p. 149171. en:

\section{https://doi.org/10.16890/rstpr.a7.n14.p149}

MAFFEI, Brenda. "O Mercosul e a complexidade: um estudo do caso dos pneus remoldados a partir do paradigma Sistêmico-holístico" (Tesis). Orientador: Karine de Souza Silva. Universidade Federal de Santa Catarina. Brasil, 2017. Disponible em: https://repositorio.ufsc.br/bitstream/handle/123456789/ 176736/346783. pdf?sequence=1\&isAllowed=y

MEJÍA, Orlando. "El diálogo entre tribunales: la jurisprudencia del Tribunal de Justicia de la Unión Europea como fuente de inspiración para los tribunales de los sistemas de integración latinoamericanos". Boletín Electrónico sobre Integración Regional del Centro Interuniversitario para Estudios de Integración (CIPEI) 2011.vol 1. p. 14-34 Disponible en:

http://www.boletincipei.unanleon.edu.ni/documentos/a rticulos/art1.pdf 


\section{Serie: Jurisprudencia * Laudos $\mathrm{N}^{0} 2$}

MERCOSUR. Secretaría del Mercosur. Laudos, Aclaraciones y Opiniones consultivas de los Tribunales del Mercosur. Montevideo: Secretaría del Mercosur,2007

PASTORI FILLOL, Alejandro. "Comentarios al primer laudo del Tribunal Permanente de Revisión del Mercosur sobre la aplicación en exceso de medidas compensatorias". EN: DRNAS de CLEMENT, Zlata (Coordinadora). Mercosur y Unión Europea. Buenos Aires: Lerner, 2008. p.23-43

PÉREZ, Adrián. "Obstáculos y desafíos del Sistema de solución de Controversias en el Mercosur (1991-2010) (Tesis). Buenos Aires: Universidad de Buenos Aires. Facultad de Ciencias Económicas. Escuela de Estudios de Posgrados. Disponible en: http://bibliotecadigital.econ.uba.ar/download/tpos/1502 -0184 PerezA.pdf

PEROTTI, Alejandro. ¿Quién paga los costos del incumplimiento de las sentencias del Tribunal Permanente de Revisión (Mercosur)? Responsabilidad del estado por violación del derecho de la integración. Revista Quaestio luris, 2011, Vol 4, №1. Disponible en: DOI: http://dx.doi.org/10.12957/rqi.2011.10195

REYES TAGLE, Yovana. "El impacto de la jurisprudencia del Tribunal de Justicia de la Unión Europea en la definición del principio de libre circulación de mercancías en la Comunidad Andina y el Mercosur". Agenda internacional, 2018, Año XXV N ${ }^{\circ}$ 36, p. 235-256. Disponible en: https://doi.org/10.18800/agenda.201801.012

SCOTTI, Luciana B. Cumplimiento e implementación de los laudos en el Mercosur. En. NEGRO, Sandra (coord.) Número especial sobre solución de diferencias 
Serie: Jurisprudencia * Laudos $\mathrm{N}^{\circ 2}$

e implementación en procesos de integración regional de Jurisprudencia Argentina (JA), tomo IV, fascículo 9. Ed. Abeledo Perrot, 2013. Disponible en: http://www.derecho.uba.ar/investigacion/investigadore s/publicaciones/scotti-cumplimiento-eimplementacion-de-laudos-mercosur-para-ja.pdf 
